\title{
Utilization of different MurNAc sources by the oral pathogen Tannerella forsythia and role of the inner membrane transporter AmpG
}

Valentina M. T. Mayer ${ }^{1}$, Markus B. Tomek ${ }^{1}$, Rudolf Figl' ${ }^{2}$, Marina Borisova ${ }^{3}$, Isabel Hottmann ${ }^{3}$, Markus Blaukopf ${ }^{4}$, Friedrich Altmann ${ }^{2}$, Christoph Mayer ${ }^{3^{*}}$ and Christina Schäffer ${ }^{1 *}$ (D)

\begin{abstract}
Background: The Gram-negative oral pathogen Tannerella forsythia strictly depends on the external supply of the essential bacterial cell wall sugar N-acetylmuramic acid (MurNAc) for survival because of the lack of the common MurNAc biosynthesis enzymes MurA/MurB. The bacterium thrives in a polymicrobial biofilm consortium and, thus, it is plausible that it procures MurNAc from MurNAc-containing peptidoglycan (PGN) fragments (muropeptides) released from cohabiting bacteria during natural PGN turnover or cell death. There is indirect evidence that in T. forsythia, an AmpG-like permease (Tanf_08365) is involved in cytoplasmic muropeptide uptake. In E. coli, AmpG is specific for the import of Nacetylglucosamine (GlcNAc)-anhydroMurNAc(-peptides) which are common PGN turnover products, with the disaccharide portion as a minimal requirement. Currently, it is unclear which natural, complex MurNAc sources T. forsythia can utilize and which role AmpG plays therein.

(Continued on next page)
\end{abstract}

\footnotetext{
* Correspondence: christoph.mayer@uni-tuebingen.de;

christina.schaeffer@boku.ac.at

${ }^{3}$ Department of Biology, Eberhard Karls Universität Tübingen, Microbiology/

Glycobiology, Interfaculty Institute of Microbiology and Infection Medicine

Tübingen, Tübingen, Germany

'Department of NanoBiotechnology, NanoGlycobiology unit, Universität für Bodenkultur Wien, Vienna, Austria

Full list of author information is available at the end of the article
}

(c) The Author(s). 2020 Open Access This article is licensed under a Creative Commons Attribution 4.0 International License, which permits use, sharing, adaptation, distribution and reproduction in any medium or format, as long as you give appropriate credit to the original author(s) and the source, provide a link to the Creative Commons licence, and indicate if changes were made. The images or other third party material in this article are included in the article's Creative Commons licence, unless indicated otherwise in a credit line to the material. If material is not included in the article's Creative Commons licence and your intended use is not permitted by statutory regulation or exceeds the permitted use, you will need to obtain permission directly from the copyright holder. To view a copy of this licence, visit http://creativecommons.org/licenses/by/4.0/ The Creative Commons Public Domain Dedication waiver (http://creativecommons.org/publicdomain/zero/1.0/) applies to the data made available in this article, unless otherwise stated in a credit line to the data. 
(Continued from previous page)

Results: We performed a screen of various putative MurNAc sources for T. forsythia mimicking the situation in the natural habitat and compared bacterial growth and cell morphology of the wild-type and a mutant lacking AmpG (T. forsythia $\Delta a m p G)$. We showed that supernatants of the oral biofilm bacteria Porphyromonas gingivalis and Fusobacterium nucleatum, and of E. coli $\triangle a m p G$, as well as isolated PGN and defined PGN fragments obtained after enzymatic digestion, namely GlcNAc-anhydroMurNAc(-peptides) and GlcNAc-MurNAc(-peptides), could sustain growth of T. forsythia wildtype, while T. forsythia $\Delta a m p G$ suffered from growth inhibition. In supernatants of $T$. forsythia $\Delta a m p G$, the presence of GlcNAc-anhMurNAc and, unexpectedly, also GlcNAc-MurNAc was revealed by tandem mass spectrometry analysis, indicating that both disaccharides are substrates of AmpG. The importance of AmpG in the utilization of PGN fragments as MurNAc source was substantiated by a significant ampG upregulation in T. forsythia cells cultivated with PGN, as determined by quantitative real-time PCR. Further, our results indicate that PGN-degrading amidase, lytic transglycosylase and muramidase activities in a T. forsythia cell extract are involved in PGN scavenging.

Conclusion: T. forsythia metabolizes intact PGN as well as muropeptides released from various bacteria and the bacterium's inner membrane transporter AmpG is essential for growth on these MurNAc sources, and, contrary to the situation in E. coli, imports both, GlcNAc-anhMurNAc and GlcNAc-MurNAc fragments.

Keywords: Tannerella forsythia, Peptidoglycan, Muropeptides, AmpG permease, N-acetylmuramic acid sources, Anhydro$\mathrm{N}$-acetylmuramic acid, Oral biofilm

\section{Background}

Tannerella forsythia is a Gram-negative, obligate anaerobic bacterium inhabiting the oral cavity that is strongly associated with periodontitis (gum disease) [1], a multifactorial, inflammatory biofilm disease affecting the tissues supporting the teeth, ultimately leading to tooth loss [2]. The disease is widespread among the adult population worldwide and its cost estimate was up to 396 billion USD in 2015 [3]. There is also mounting evidence of a link between periodontitis and various systemic medical conditions, including, e.g., cardiovascular diseases, rheumatoid arthritis, and Alzheimer's disease [4,5]. T. forsythia is usually found alongside the Gram-negatives Porphyromonas gingivalis and Treponema denticola, together constituting the "red complex" of periodontal pathogens and acting as late colonizers of a dysbiotic polymicrobial biofilm, which causes periodontitis $[6,7]$. Other crucial colonizers of this oral biofilm (commonly known as dental plaque) are streptococci adhering to mucosal surfaces and the "bridge organism" Fusobacterium nucleatum [8].

Among the dental plaque bacteria, $T$. forsythia has a unique status, because of its auxotrophy for the commonly essential bacterial peptidoglycan (PGN) cell wall sugar $N$ acetylmuramic acid (MurNAc) and, thus, strictly depends on external supply of MurNAc for growth and maintenance of cell morphology, when grown in monospecies laboratory culture [9]. We recently confirmed by scanning electron microscopy the transition from healthy, rodshaped to fusiform $T$. forsythia cells upon stepwise MurNAc depletion [10]. It is important to consider that growth in monospecies culture does not reflect the situation in the oral cavity, where $T$. forsythia is part of the polymicrobial biofilm consortium. Recently it was found that a pool of muropeptides from $F$. nucleatum could replace free MurNAc to promote growth of $T$. forsythia [11].

T. forsythia's MurNAc auxotrophy is due to the absence of the genes for the PGN biosynthesis enzymes MurA and MurB in its genome [12]; these are required for the de novo synthesis of uridyl-diphosphate (UDP) activated MurNAc for subsequent channelling into the PGN biosynthesis route [13]. PGN is a bag-shaped macromolecule (PGN sacculus) external to the bacterial cytoplasmic membrane that serves as a protection from adverse environmental effects and disruption due to high internal osmotic pressure and, thus, is essential for bacterial survival [14]. Despite its predictably unique PGN metabolism, $T$. forsythia possesses a common Gram-negative PGN structure of the A1 $\gamma$-type [15] as was recently published by our group [16]: MurNAc and $\beta$-1,4-linked GlcNAc alternatingly form glycan backbone strands, terminating with a non-reducing 1,6-anhydroMurNAc residue (anhMur$\mathrm{NAc}$ ) at the reducing end, with stem peptides composed of alanine (Ala), glutamic acid (Glu), meso-diaminopimelic acid ( $m$-DAP), and Ala, and likely a direct cross-linkage between $m$-DAP on the third and Ala on the fourth position of cross-linked stem peptides. This PGN type occurs also in $P$. gingivalis [16] and Escherichia coli [13], while in the PGN of $F$. nucleatum, $m$-DAP is replaced by lanthionine [17].

In the course of the natural cell wall turnover, bacteria enzymatically digest their PGN [18]. The PGN backbone may be cleaved by $N$-acetylmuramidases cutting the $\beta-1$, 4 glycosidic bond between MurNAc and GlcNAc, and lytic transglycosylases, which concomitantly introduce a 1,6-anhydro bond at the MurNAc residue [13, 19]. Endo-acting $N$-acetylglucosaminidases catalyse the hydrolysis of the other glycosidic bond (GlcNAc- $\beta-1,4-$ 
MurNAc), thus, generating distinct MurNAc-GlcNAcpeptides [20]. Accrued PGN fragments (muropeptides) are either recycled or released into the environment, where they are thought to get accessible for cohabiting bacteria [11].

For PGN turnover and recycling [21], many Gramnegatives involve lytic transglycosylases generating GlcNAc-anhMurNAc-peptides (anhydromuropeptides) and (AmpG-like) permeases to transport these anhydromuropeptides across the cytoplasmic membrane. AmpG was originally identified in $E$. coli as a regulator necessary for the induction of the $\beta$-lactamase AmpC [18]. Its function as a PGN-recycling permease with likely specificity for GlcNAc-anhMurNAc(-peptides) was shown by Cheng and Park [22]. The authors detected inhibition of the uptake of radiolabelled GlcNAc-anhMurNAc and GlcNAcanhMurNAc-peptides by an E. coli $\triangle a m p G$ deletion mutant in comparison to the wild-type. By adding carbonyl cyanide 3-chlorophenylhydrazone, the uptake in the wildtype was decreased to a similar level as in the mutant, showing the transporter's dependence on a proton motive force. Regarding other substrates, including GlcNAcMurNAc(-peptides) and anhMurNAc(-peptides), no significant difference was found between wild-type and the $\triangle a m p G$ mutant. The genome of $T$. forsythia encodes one muropeptide permease AmpG homologue (Tanf_08365), which displays $27 \%$ amino acid identity with the E. coli permease and is located in the middle of a nine-genes spanning genomic cluster (Tanf_08345 to Tanf_08385) representing a single transcriptional unit [11]. This gene cluster comprises several putative PGN recycling genes, including the already characterized genes for the MurNAc transporter MurT [23], the kinase MurK, and the etherase MurQ [10], which work in concert along the anabolic UDP-MurNAc recycling pathway [21]. The function of Tanf_08365 as a muropeptide transporter was recently indirectly confirmed by heterologous expression in E. coli applying a reporter system with a muropeptide inducible $\beta$-lactamase reporter gene [17]. A plasmid containing the inducible reporter gene was introduced to an E. coli double mutant lacking the muropeptide transporter AmpG and the cytosolic amidase AmpD. The complementation of this E. coli reporter strain with the T. forsythia ampG gene restored the uptake of PGN recycling products, and due to the resulting cytosolic accumulation of anhMurNAcpeptides, induction of $\beta$-lactamase activity could be measured. Furthermore, a direct regulation of the implied operon by the hybrid two component system GppX was revealed, since the expression of GppX (Tanf_13760) led to a seven-fold higher induction of the promotor region of the operon and Tanf_08365 was significantly downregulated in a $T$. forsythia $g p p X$ deletion mutant $[11,24]$. Notably, growth of the gppX mutant was not completely abolished on either muropeptides or free
MurNAc, suggesting that the $a m p G$-containing operon is expressed constitutively at basal levels [11].

In this present study, various MurNAc sources of different complexity likely present in the polymicrobial biofilm setting in the oral habitat were analysed for their suitability to sustain growth of the periodontal pathogen $T$. forsythia. Further, the role and specificity of the AmpG permease in the utilization of these putative MurNAc substitutes was assessed. Specifically, this included (i) preparation of MurNAc sources from oral bacteria and from $E$. coli, followed by compositional analysis by HPAEC-PAD, electrospray ionization LC-ESI-MS, and $\mathrm{MS}^{2}$, (ii) chemical synthesis of anhMurNAc, (iii) monitoring of growth and cell morphology using SEM, as well as (iv) comparing the effects of the different MurNAc sources in T. forsythia wild-type versus a novel isogenic $T$. forsythia $\triangle a m p G \mathrm{mu}-$ tant to unravel the role and specificity of the cytoplasmic membrane transporter AmpG in the PGN metabolism. Overall, this study sheds light on the metabolic strategies the periodontal pathogen $T$. forsythia has elaborated to build up its cell wall PGN.

\section{Results}

\section{Preparation and analysis of putative MurNAc sources}

To obtain insight into which complex MurNAc sources likely present in the oral habitat $T$. forsythia can metabolize to compensate for its MurNAc auxotrophy, different MurNAc sources mimicking the circumstances in the oral biofilm were prepared and their composition was analysed.

Considering $T$. forsythia as part of the oral biofilm consortium, supernatants from the red complex member $P$. gingivalis W83 and the "bridge organism" F. nucleatum OMZ 589, as well as E. coli MC4100 and E. coli TP72 $(\triangle a m p G)$, were harvested at $\mathrm{OD}_{600} \sim 2.0$. Supernatants were analysed by LC-ESI-MS, using an amaZon ion trap mass spectrometer, and data were verified by $\mathrm{MS}^{2}$ analysis, using standards for MurNAc and anhMurNAc.

While no PGN fragments were detected in supernatants of E. coli MC4100 indicative of efficient PGN recycling, the supernatant of the E. coli $\triangle a m p G$ mutant, which is unable to take up its PGN recycling products due to deficiency in $\operatorname{amp} G$, was confirmed to contain GlcNAcanhMurNAc, as published previously (Supplemental Fig. S1A) [25]. In supernatants of $F$. nucleatum OMZ 589, MS analysis revealed the presence of GlcNAc-anhMurNAc indicative of inefficient $\mathrm{PGN}$ recycling. Conforming with the theoretical mass of $m / z 479.19$, the disaccharide was observed with a mass of $m / z 479.29[\mathrm{M}+\mathrm{H}]^{+}$and verified by $\mathrm{MS}^{2}$ analysis (Supplemental Fig. S1B). The analysis of the $P$. gingivalis supernatant revealed to be more difficult. However, after enrichment via hydrophobic interaction liquid chromatography-solid phase extraction (HILIC-SPE), the presence of GlcNAc-MurNAc could be detected. Conforming with the theoretical mass of $\mathrm{m} / \mathrm{z} 497.19$, the 
disaccharide was observed with a mass of $\mathrm{m} / \mathrm{z} 497.18$ $[\mathrm{M}+\mathrm{H}]^{+}$and verified by $\mathrm{MS}^{2}$ analysis (Supplemental Fig. S1C).

PGN sacculi as a putative source of MurNAc were isolated from T. forsythia wild-type, P. gingivalis W83, F. nucleatum OMZ 589, and E. coli MC4100, following a published protocol [26]. After digestion of PGN with the $\mathrm{N}$-acetylmuramidase mutanolysin, the PGN composition was determined by LC-MS, revealing the presence of GlcNAc-MurNAc-peptides (G-M-tri/tetra) and dimers thereof as typical digestion products. For E. coli [13], as well as $T$. forsythia and $P$. gingivalis, the stem peptide is composed of Ala, Glu, $m$-DAP and Ala, referring to the PGN-type A1 $\gamma$, as published previously [16]. Regarding the PGN of $F$. nucleatum, Vasstrand et al. reported that lanthionine instead of $m$-DAP occurs in the stem peptide $[27,28]$. This result was confirmed by our study, providing the first MS data for F. nucleatum PGN. The observed peaks were $m / z$ 889.32 $[\mathrm{M}+\mathrm{H}]^{+}$for G-M-tri and $m / z$ $960.36[\mathrm{M}+\mathrm{H}]^{+}$for G-M-tetra, and, thus, in accordance with theoretical masses of $\mathrm{m} / z 889.33$ and $\mathrm{m} / z 960.37$ for lanthionine containing fragments (Supplemental Fig. S2).

The preparation of GlcNAc-MurNAc-peptides and GlcNAc-anhMurNAc-peptides was accomplished by digesting E. coli MC4100 PGN with mutanolysin and the lytic transglycosylase Slt70, respectively. Additional treatment of the digestion products with the amidase AmiD produced free disaccharides as confirmed by LCMS (Supplemental Fig. S3), in addition to peptides.

Finally, anhMurNAc was chemically synthesized [29] and purified by HILIC, with an achieved overall yield of 5.4\%. All analytical NMR data of anhMurNAc were in agreement with previously published data (Supplemental Fig. S4).

\section{Identification of MurNAc sources for T. forsythia}

The different putative MurNAc sources of known composition as determined above were added as a BHI medium supplement for $T$. forsythia wild-type and $T$. forsythia $\triangle a m p G$, instead of free MurNAc. Of note, T. forsythia is routinely grown with supplementation of $10 \mu \mathrm{g} \mathrm{ml}^{-1}$ Mur$\mathrm{NAc}$, reaching a maximal $\mathrm{OD}_{600}$ of $\sim 1.6$, while supplementation of $1 \mu \mathrm{g} \mathrm{ml}^{-1}$ MurNAc supports growth up to an $\mathrm{OD}_{600}$ of $\sim 1.0$ only (Fig. 1a). To correct for differences in the MurNAc concentration of the different sources, supernatants as well as PGN sacculi were hydrolysed and the MurNAc concentration was determined by HPAEC-PED. Since standards for MurNAc and anhMurNAc had the same retention time of $42.4 \mathrm{~min}$, a combined value for both components was obtained (data not shown).

First, MurNAc was substituted by bacterial culture supernatants. Due to the low detectable amount of MurNAc/ anhMurNAc in bacterial supernatants of $F$. nucleatum, $P$. gingivalis and $E$. coli, these were added in the most concentrated form possible (before reaching viscosity), equalling a
MurNAc/anhMurNAc concentration in the growth medium below $1 \mu \mathrm{g} \mathrm{ml}^{-1}$. While the supply of the GlcNAcanhMurNAc-containing supernatant of E. coli $\triangle a m p G$ yielded a $T$. forsythia culture of an $\mathrm{OD}_{600}$ of $\sim 0.8$, supernatants of $E$. coli wild-type could not sustain growth of T. forsythia wild-type due to the absence of any detectable muropeptides. Supernatants of $F$. nucleatum, also shown to contain GlcNAc-anhMurNAc, albeit in lower concentration (Supplemental Fig. S1), sustained growth of T. forsythia wild-type to an $\mathrm{OD}_{600}$ of $\sim 0.6$ and supernatants of $P$. gingivalis, containing minimal amounts of GlcNAc-MurNAc, sustained growth to an $\mathrm{OD}_{600}$ of $\sim 0.45$ (Fig. 1b).

Growth of T. forsythia wild-type with isolated PGN sacculi corresponding to a final MurNAc/anhMurNAc concentration in the growth medium of $1 \mu \mathrm{g} \mathrm{ml}^{-1}$ was unrestrictedly sustained, independent of the bacterial source from which it was isolated from. Cultures yielded $\mathrm{OD}_{600}$ values of $\sim 1.4$ (Fig. 1c) and bacterial cells showed no alteration of cell morphology, according to SEM evidence (Fig. 2). The supply of specific digests of PGN by the $N$ acetylmuramidase mutanolysin, containing mainly GlcN Ac-MurNAc-peptides, had a similar effect on growth of $T$. forsythia, reaching $\mathrm{OD}_{600}$ values of $\sim 1.3$. In comparison, digests of PGN by the lytic transglycosylase Slt70, containing mainly GlcNAc-anhMurNAc-peptides, restricted growth of T. forsythia wild-type to an $\mathrm{OD}_{600}$ of $\sim 0.8$. Additional treatment of PGN digests with amidase AmiD, producing free disaccharides and peptides, resulted in a comparable growth behaviour according to $\mathrm{OD}_{600}$ measurement of $T$. forsythia (Fig. 1d).

Based on the demonstrated suitability of GlcNAcanhMurNAc as a MurNAc source for T. forsythia wildtype (Fig. 1d), also the effect of synthesized anhMurNAc at a concentration of $10 \mu \mathrm{g} \mathrm{ml}^{-1}$ on bacterial growth was investigated. Unexpectedly, also anhMurNAc alone supported growth of T. forsythia wild-type, however, it constitutes a rather poor substrate with a reproducibly maximal $\mathrm{OD}_{600}$ of $\sim 0.32$ (Fig. 1a).

\section{Digestion of PGN by $T$. forsythia cell extracts}

To obtain first insight into the enzymatic activities $T$. forsythia might require for PGN scavenging, a T. forsythia cell extract was used as a putative source of such enzymes and digestion products of $E$. coli PGN after treatment with the extract were determined. For this purpose, T. forsythia wild-type was cultivated until the late exponential growth phase, either grown with free MurNAc or with $E$. coli PGN as a MurNAc source since we reasoned that PGNactive enzymes might be induced upon growth on PGN. Subsequently, cells were lysed and harvested by ultracentrifugation to prepare enzyme-rich extracts. Finally, a preparation of $E$. coli $\mathrm{PGN}$ was incubated with the extract overnight at $37^{\circ} \mathrm{C}$ and aliquots were analysed by LC-MS after several time points. We found that a cell extract of $T$. 

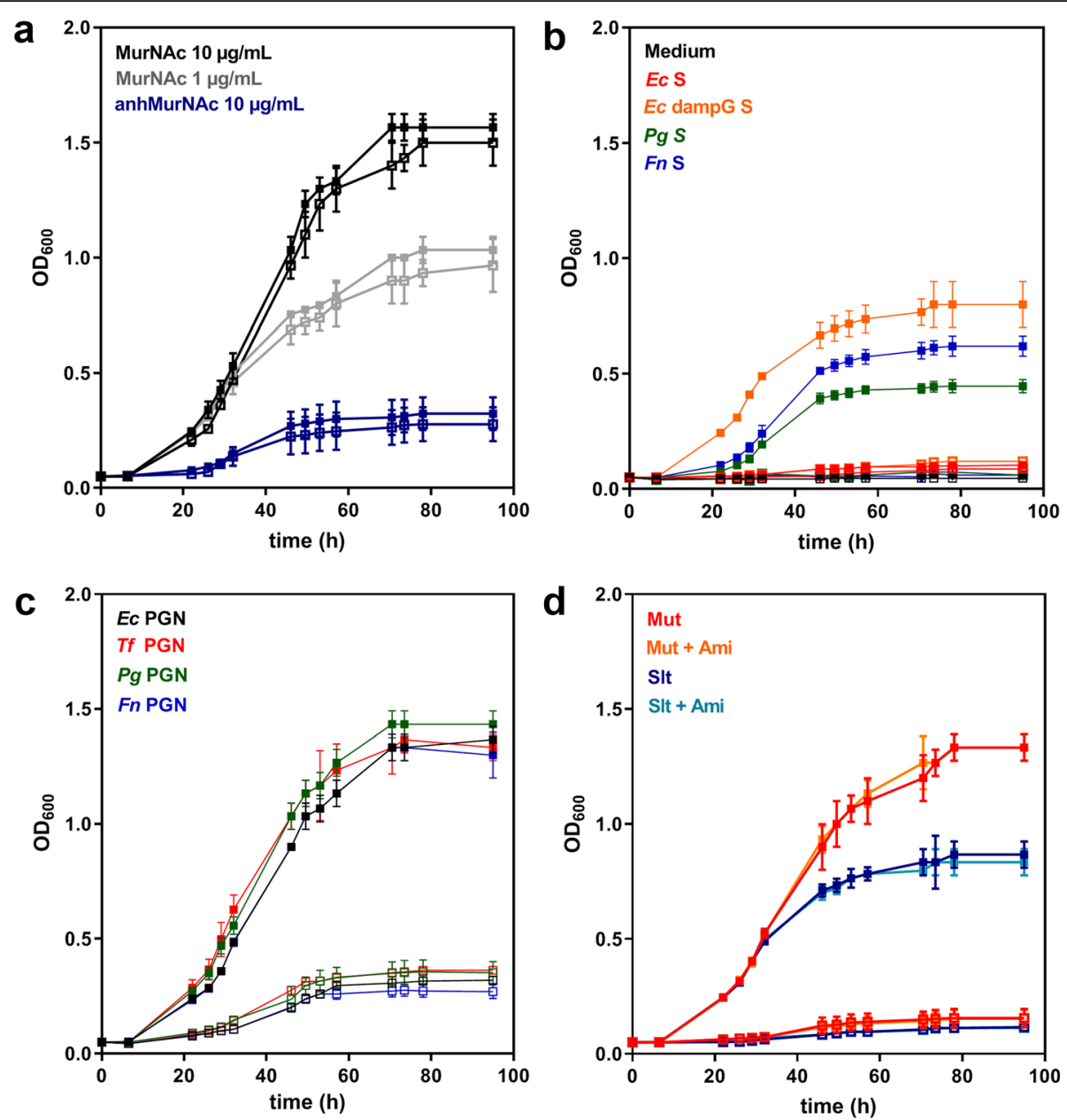

\section{Tf \\ Tf $\Delta a m p G$}

Fig. 1 Growth of T. forsythia wild-type (Tf; solid symbols) and T. forsythia $\triangle a m p G$ mutant (Tf $\triangle a m p G$; open symbols) with MurNAc and anhMurNAc (a) and different MurNAc substituents (color-coded), including supernatants of E. coli MC4100, E. coli $\triangle$ ampG, P. gingivalis W83 and F. nucleatum OMZ 589 (b); PGN of E. coli, T. forsythia, P. gingivalis and F. nucleatum (c); and digests of E. coli PGN with mutanolysin (Mut), yielding GlcNAc-MurNAc-peptides, and Slt70 (Slt), yielding GlcNAc-anhMurNAc-peptides, plus AmiD (+Ami), cutting off the peptides, yielding GlcNAcMurNAc (d). The mean $\mathrm{OD}_{600}$ values of biological triplicates and standard errors are shown

forsythia successfully digested PGN, revealing the disaccharides GlcNAc-MurNAc and GlcNAc-anhMurNAc as well as MurNAc as predominant digestion products in addition to a small amount of anhMurNAc, and, plausibly, peptides of different length (not shown), which all increased over the analysed incubation time of $20 \mathrm{~h}$ (Fig. 3a, Supplemental Fig. S5). No difference in digestion products was evident between cultivation of T. forsythia with MurNAc versus PGN, however, the amount of detected products was higher when using a cell extract prepared from cultures grown with PGN; this was especially true for GlcNAc-MurNAc and GlcNAc-anhMurNAc, which increased by 3.8 -fold and 5.3-fold, respectively, with GlcNAc-MurNAc being the major digestion product in absolute number occurring in an 8.6-fold excess compared to GlcNAc-anhMurNAc. GlcNAc-MurNAc was the major disaccharide digestion product obtained regardless of the MurNAc source used for the preparation of the cell extract; this was unambiguously confirmed based on the successful digestion with the $\mathrm{N}$-acetylglucosaminidase NagZ [20] (Fig. 3b).

\section{Role and specificity of $T$. forsythia AmpG in the uptake of MurNAc substitutes}

To elucidate the role of the muropeptide transporter AmpG in uptake of MurNAc substitutes, an isogenic mutant of $T$. forsythia was constructed by homologous recombination ( $T$. forsythia $\triangle a m p G$ ) (Supplemental Fig. S6) and cultivated 


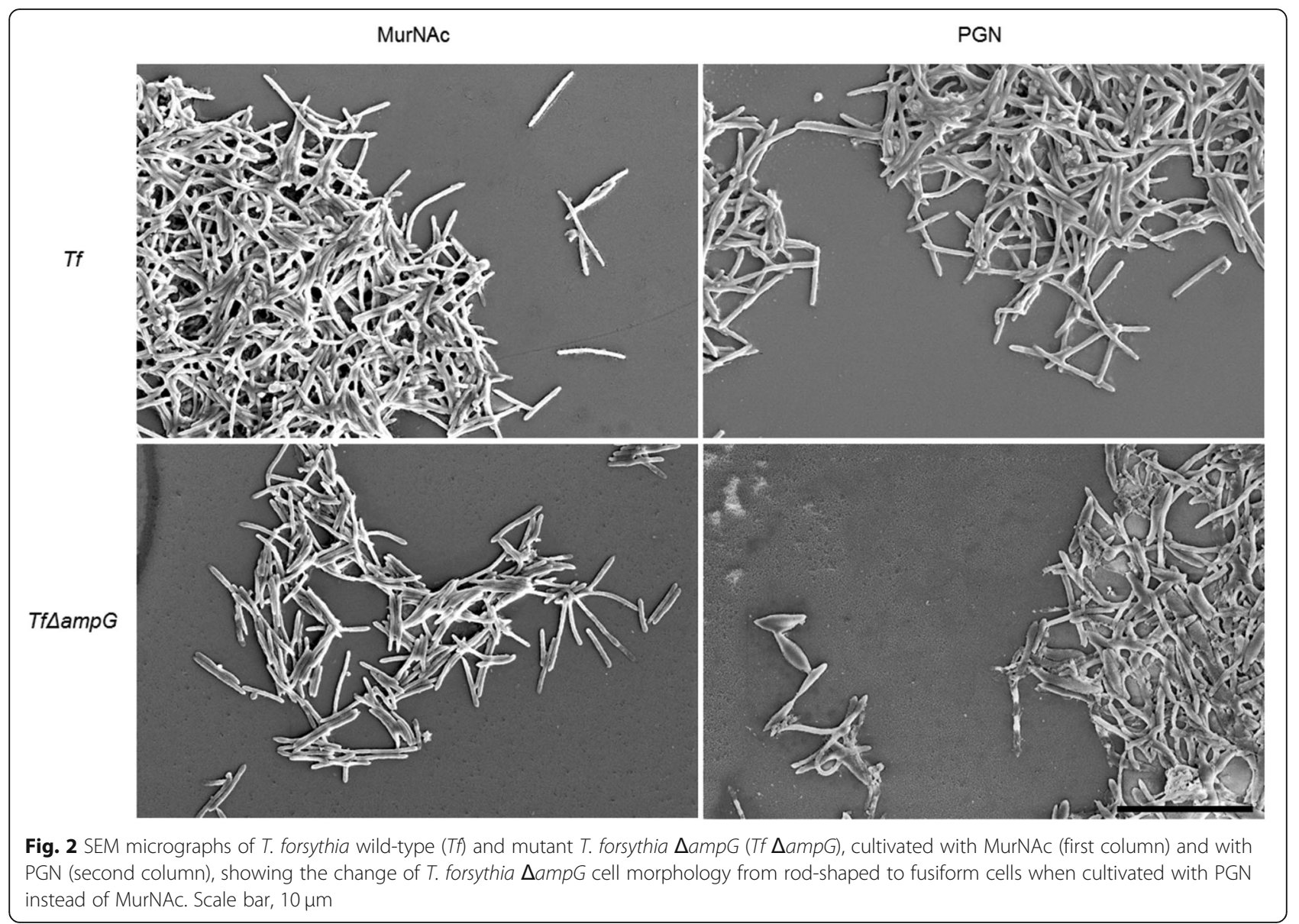

with the aforementioned MurNAc sources. In comparison to T. forsythia wild-type, T. forsythia $\triangle a m p G$ suffered from severe growth impairment, when cultivated with alternate MurNAc sources. While T. forsythia $\triangle a m p G$ provided with $10 \mu \mathrm{g} \mathrm{ml}^{-1}$ MurNAc yielded an $\mathrm{OD}_{600}$ of 1.5 (Fig. 1a) supportive of the fact that MurNAc is imported primarily via MurT [23] and not via AmpG - the cultivation with supernatants of the cohabiting bacteria $F$. nucleatum and $P$. gingivalis, as well as E. coli $\triangle a m p G$ led to growth inhibition (Fig. 1b). Substitution of MurNAc by undigested PGN enabled restricted growth of $T$. forsythia $\triangle a m p G$ to an $\mathrm{OD}_{600}$ of $\sim 0.3$ (Fig. 1c), indicating that the majority of processed PGN is imported via AmpG; the residual growth might be an effect of free MurNAc, which we showed to be a product of PGN digestion by a $T$. forsythia extract (compare with Fig. 3a). Concomitantly, SEM demonstrated the change of T. forsythia $\triangle a m p G$ cells from rod-shaped to fusiform morphology, as is typical under MurNAc starvation (Fig. 2). The supply of specific PGN digests also resulted in growth inhibition of T. forsythia $\triangle a m p G$. Surprisingly, not only a PGN digest with Slt70, containing mainly GlcNAcanhMurNAc(-peptides) - the preferred substrates of E. coli AmpG [22] - led to growth inhibition, but also digests with mutanolysin, containing GlcNAc-MurNAc(-peptides) failed in sustaining growth of T. forsythia $\triangle a m p G$ (Fig. 1d).

Substitution of MurNAc by anhMurNAc restricted growth of $T$. forsythia $\triangle a m p G$ to an $\mathrm{OD}_{600}$ of $\sim 0.28$, which is comparable to the growth of the wild-type upon provision of anhMurNAc, ruling out the involvement of AmpG in the uptake of anhMurNAc (Fig. 1a).

\section{Determination of relative expression level of AmpG}

The direct contribution of the T. forsythia inner membrane transporter AmpG (Tanf_08365) in the utilization of PGN as a MurNAc source by the pathogen was supported by determining the relative expression level of AmpG under PGN supplementation in comparison to free MurNAc. RNA was isolated from T. forsythia wild-type cultivated with either MurNAc or E. coli PGN, transcribed into cDNA, and the relative expression level of AmpG was determined by qPCR. When the AmpG expression level in cultures grown with MurNAc until the exponential growth phase was normalized to 1 , the relative expression level in the stationary phase was 0.56 . In contrast, when cultivated with PGN, the relative expression level was 4.19 in the exponential and 2.14 in the stationary growth phase. 
In conclusion, when T. forsythia was cultivated with PGN, AmpG was upregulated approximately 4.1-fold in the exponential and 3.8-fold in the stationary growth phase (Fig. 4). Further, the data supports a higher AmpG import rate of PGN fragments during the exponential growth phase, which conforms with previous observations made by others in the context of $E$. coli PGN recycling involving the AmpG permease [22].

\section{PGN fragments in the supernatant of $T$. forsythia $\triangle a m p G$}

Since an E. coli mutant lacking the inner membrane muropeptide transporter AmpG was shown to release GlcNAcanhMurNAc into the medium [22], supernatants of T. forsythia $\triangle a m p G$ were analysed regarding the accumulation of PGN fragments. T. forsythia wild-type and T. forsythia $\triangle a m p G$ were cultivated under standard conditions and supernatants were harvested in the stationary growth phase. LC-ESI-MS measurements revealed the presence of the disaccharides GlcNAc-anhMurNAc as well as GlcNAcMurNAc, only for the mutant T. forsythia $\triangle a m p G$. Observed masses of $\mathrm{m} / z$ 479.22 $[\mathrm{M}+\mathrm{H}]^{+}$for GlcNAcanhMurNAc and $m / z$ 497.19 $[\mathrm{M}+\mathrm{H}]^{+}$for GlcNAcMurNAc were in accordance with the theoretical masses of $m / z 479.19$ and $m / z 497.20$, respectively, and confirmed by $\mathrm{MS}^{2}$ analysis (Fig. 5).

\section{Discussion}

Periodontitis is a polymicrobial disease, in which oral biofilm bacteria organize to distribute metabolic activities for increasing their fitness [30]. It is the complexity of bacterial community interactions that is decisive for bacterial dysbiosis and, consequently, the etiology of the disease. This study
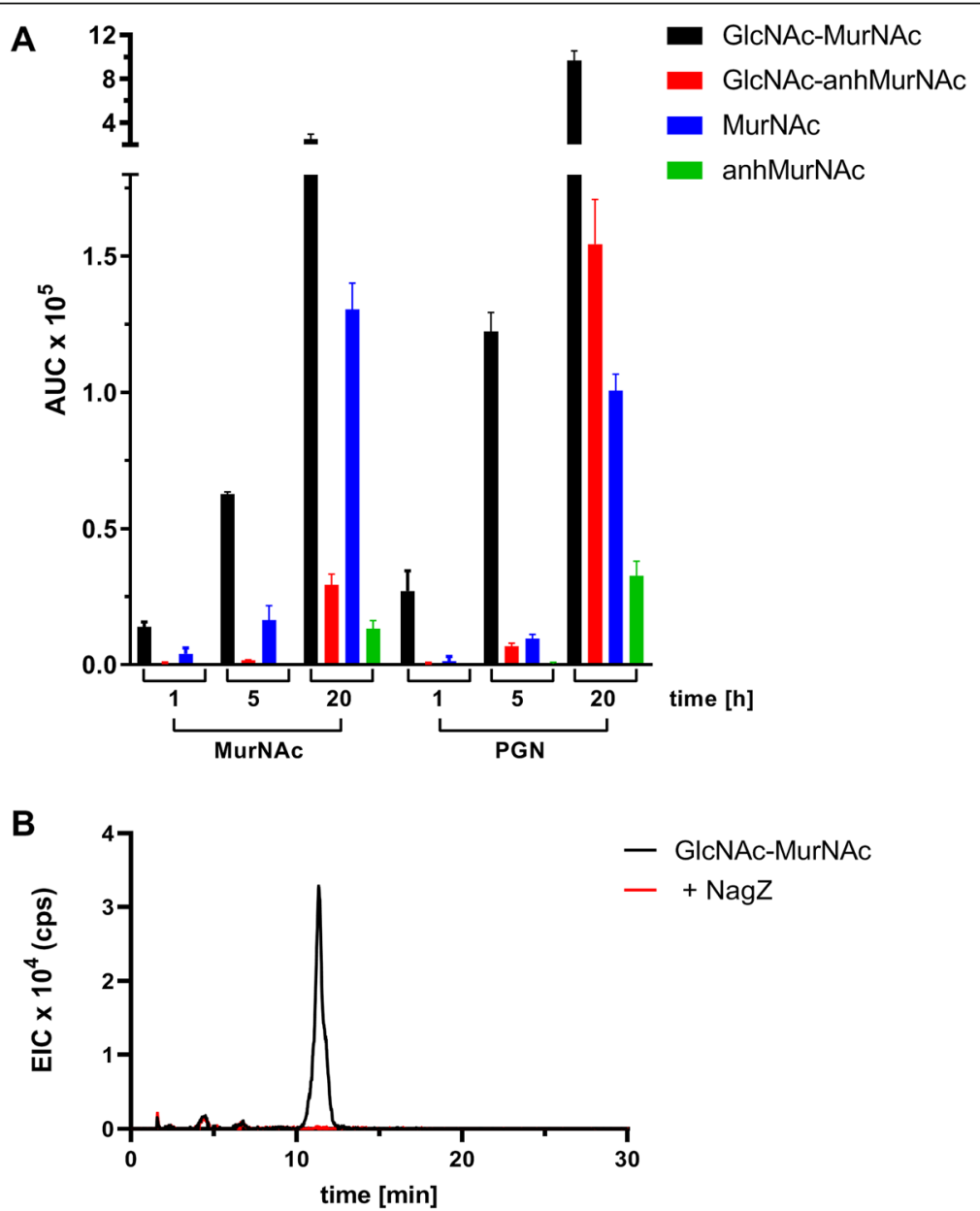

Fig. 3 Digestion products obtained upon incubation of E. coli PGN with cell extracts of T. forsythia, prepared from cultures grown with MurNAc or PGN. Samples were taken after several time points and analysed by LC-ESI-MS in negative ion mode, showing the presence of GICNAc-MurNAc - $m / z$ $495.18[\mathrm{M}-\mathrm{H}]^{-}$, GlcNAc-anhMurNAc - m/z 477.17 [M-H] $]^{-}$, MurNAc - m/z $292.11[\mathrm{M}-\mathrm{H}]^{-}$and anhMurNAc - m/z 274.09 [M-H] $]^{-}$. Measured masses were in accordance with the calculated masses of $\mathrm{m} / \mathrm{z} 495.18, \mathrm{~m} / \mathrm{z} 477.17, \mathrm{~m} / \mathrm{z} 292.10$, and $\mathrm{m} / \mathrm{z} 274.09$, respectively. Shown are the mean values of triplicates calculated from the area under the curve (AUC) (a). The disaccharide was confirmed to be GlcNAc-MurNAc based on the successful digestion with the $\mathrm{N}$-acetylglucosaminidase NagZ, shown as the extracted ion chromatogram (EIC) upon elution from a SeQuant ${ }^{\oplus}$ ZIC $^{\oplus}$-pHILIC column, before (black) and after (red) enzyme digestion (b) 
sought to investigate how the periodontal pathogen $T$. forsythia can benefit from cohabiting biofilm bacteria to overcome its MurNAc auxotrophy. T. forsythia possesses a conventional Gram-negative PGN of the A1 $\gamma$-type [16]; thus, it is conceivable to assume that the bacterium procures the essential PGN building block MurNAc from the oral biofilm consortium. Currently it is unknown, which sources of MurNAc likely available in the oral cavity $T$. forsythia can access. Identifying these sources and understanding how they are imported via the bacterial membrane barriers and metabolized by $T$. forsythia does not only constitute a necessary step for understanding how this pathogen thrives in the oral habitat but can also inform about novel strategies to combat periodontitis, for which no targeted treatment is currently available.

During their life cycle, oral biofilm bacteria - as any kind of bacteria - degrade their PGN cell wall by specific sets of autolytic enzymes and release PGN fragments into the environment; in parallel, they recycle their cell wall turnover products using different strain-specific routes [21]. For $T$. forsythia, recently an inner membrane transporter MurT dedicated to the import of free MurNAc was identified constituting a novel transporter family conserved within the Bacteroidetes phylum, to which T. forsythia is affiliated [23]. However, it is unclear where the transporter's MurNAc substrate is derived from. Once imported into the cytoplasm of T. forsythia, the path of MurNAc can take two branches. One channels MurNAc into PGN biosynthesis (anabolic route), following conversion into UDP-MurNAc via MurNAc-1P by the subsequent action of the enzymes AmgK and MurU [21] - thereby likely replacing the MurA/ $\mathrm{B}$ pathway commonly employed by bacteria to generate UDP-MurNAc from UDP-GlcNAc - while the MurKkinase/MurQ-etherase pathway displays a MurNAc catabolic route generating GlcNAc-6P from MurNAc via MurNAc-6P, which is further shuttled into glycolysis [10]. We have recently shown that deletion of T. forsythia's murK gene blocks MurNAc catabolism and allows the direction of MurNAc to PGN biosynthesis, resulting in a growth advantage of the bacterium in MurNAc-depleted medium [10].

To shed light on T. forsythia's MurNAc sources, we have chosen $P$. gingivalis and $F$. nucleatum as plausible providers of MurNAc sources, both of which are long time-established members of the oral biofilm consortium and known to associate with T. forsythia [31]. For comparison, non-oral $E$. coli was included in our study.

Culture supernatants of both $P$. gingivalis and $F$. nucleatum were suitable to sustain growth of T. forsythia wildtype (Fig. 1b). Interestingly, GlcNAc-MurNAc was identified as the MurNAc source present in P. gingivalis, while GlcNAc-anhMurNAc was found in F. nucleatum (Supplemental Fig. S1). This was supported by using the supernatant of an E. coli $\triangle a m p G$ mutant that is known to release the GlcNAc-anhMurNAc disaccharide [25].
Noticeably, MS analysis of supernatants was first considered impossible, since the cultivation medium constitutes an extremely complex matrix. Tedious optimisation efforts and application of $\mathrm{MS}^{2}$ analysis finally allowed a reproducible detection of small amounts of PGN fragments in the massive noise of supernatants. When comparing maximally reachable $\mathrm{OD}_{600}$ values of the $T$. forsythia cultures upon supplying the different bacterial supernatants, it needs to be stressed that while supernatants of the different bacterial cultures were harvested at the same $\mathrm{OD}_{600}$ values, slight differences in the final MurNAc concentration applied due to the necessary concentration procedure. In silico analyses showed that the genomes of $P$. gingivalis and $F$. nucleatum lack enzymes dedicated to PGN recycling, such as the anomeric MurNAc/GlcNAc kinase AmgK as well as the anhMurNAc kinase AnmK catalysing the conversion from anhMurNAc into MurNAc-6P. This might explain the release of PGN fragments by these bacteria during cell wall turnover. For comparison, an E. coli supernatant which did not contain any detectable MurNAc source due to its efficient PGN recycling, could not sustain growth of T. forsythia (Fig. 1b). The utilization of GlcNAc-anhMurNAc as the sole MurNAc source might implicate that $T$. forsythia is able to utilize anhMurNAc as a MurNAc substitute, at least under the applied conditions. To follow up this consideration, growth experiments with anhMurNAc as a single sugar were performed, showing, however, only restricted growth of T. forsythia (Fig. 1a). This might be due to inefficient import of anhMurNAc or a low enzymatic metabolization rate of the compound in the cytoplasm. In E. coli, anhMurNAc can enter the cytoplasm via the phosphotransferase system-dependent MurNAc transporter MurP, which is absent in T. forsythia, where it is further phosphorylated by the anhMurNAc kinase AnmK, as shown by Uehara et al. [32]. On the other hand, the authors reported that E. coli could not grow in minimal medium supplemented with anhMurNAc, in contrast to MurNAc, and, thus, assumed that anhMurNAc might be toxic to cells and readily exported by an efflux pump, if not phosphorylated. The contribution of AmpG as an anhMurNAc transporter in T. forsythia cells could be ruled out in our study, since growth of T. forsythia $\triangle a m p G$ upon provision of anhMurNAc was restricted to a similar level as the wild-type (Fig. 1a). While T. forsythia's MurNAc transporter MurT could be considered as a reasonable transporter for anhMurNAc, the subsequent metabolic strategy remains to be explored, since in the T. forsythia genome, no AnmK ortholog for conversion of anhMurNAc into MurNAc-6-phosphate is predicted.

Furthermore, we showed that $T$. forsythia can metabolize intact PGN, regardless of the PGN type (i.e., $\mathrm{A} 1 \gamma$ in the case of $P$. gingivalis and $E$. coli, and $\mathrm{A} 1 \delta$ in the case of $F$. nucleatum) to cover its MurNAc requirements (Fig. 1c). We conclude that $T$. forsythia expresses 


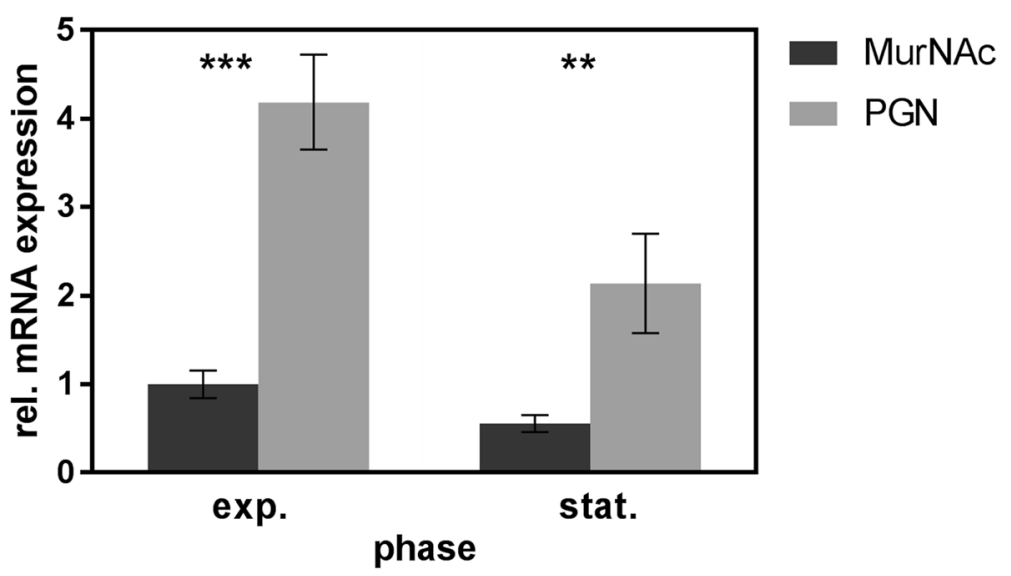

Fig. 4 Relative mRNA levels of ampG in T. forsythia wild-type cultures grown with MurNAc (black) or PGN (grey) until the exponential and stationary growth phase, respectively, as determined by PPCR. Standard errors of triplicates are shown and asterisks indicate significant differences between samples, determined by the unpaired Student's t-test $\left.{ }^{* *} P \leq 0.01,{ }^{* * *} P \leq 0.001\right)$

an active muramidase and a lytic transglycosylase for processing non-native PGN for subsequent recycling, since a T. forsythia cell extract yielded GlcNAcMurNAc, GlcNAc-anhMurNAc as well as MurNAc and anhMurNAc as digestion products from E. coli PGN (Fig. 3a, Supplemental Fig. S5). Thus, T. forsythia seems to mainly scavenge PGN sugar backbone fragments, most likely generated from muropeptides upon cleavage of peptides by an active amidase, for building up its cell wall PGN; this is supported by the fact that $F$. nucleatum PGN, which has a different stem peptide composition compared to T. forsythia's endogenous PGN, is also growth promoting for the bacterium.

Concerning further processing of PGN fragments in $T$. forsythia cells, the involvement of the AmpG permease Tanf_08365 in muropeptide uptake was recently shown indirectly in an E. coli host, in which AmpG-dependent accumulation of cytosolic muropeptides was evident via a muropeptide inducible $\beta$-lactamase reporter gene [11]. Within this present study, we investigated the role of Tanf08365 directly in $T$. forsythia by using an isogenic $T$. forsythia $\triangle a m p G$ mutant. Our approach also allowed us to determine the specificity of $T$. forsythia AmpG. Although we cannot rule out that $T$. forsythia AmpG may also transport (anhydro-)muropeptides, our results indicate that GlcNAcMurNAc and GlcNAc-anhMurNAc disaccharides lacking peptide substituents are the major substrates of the transporter when $T$. forsythia grows on PGN.

AmpG belongs to permeases from the major facilitator superfamily of transport proteins, which are ubiquitously present in Gram-negative bacteria [33-35]. According to the current accumulated data, the principal requirement of AmpG is for the presence of the disaccharide GlcNAcanhMurNAc. These unique substrates for AmpG, which contain murein peptides linked to GlcNAc-anhMurNAc, are produced by PGN turnover during exponential growth [22].
Our results demonstrated that Tanf_08365 is essential for growth of $T$. forsythia on complex MurNAc sources provided by other bacteria. This was supported by SEM analysis of cell morphology (Fig. 2). Growth of $T$. forsythia $\triangle a m p G$ was severely impaired when cultivated with intact PGN of cohabiting bacteria (i.e., P. gingivalis or F. nucleatum) and even completely inhibited when cultivated with supernatants (Fig. 1b, c). In comparison, upon supplementation of free MurNAc, which can enter the cytoplasm via the inner membrane transporter MurT [23], growth of T. forsythia $\triangle a m p G$ until maximally reachable $\mathrm{OD}_{600}$ was observed (Fig. 1a). Supportive of this finding, we found that the relative expression level of AmpG was significantly upregulated when T. forsythia was cultivated with PGN instead of free MurNAc (Fig. 4).

Considering that an $E$. coli PGN digest by the T. forsythia cell extract yielded GlcNAc-MurNAc, besides the classical PGN recycling product GlcNAc-anhMurNAc, it was conceivable to assume that both these disaccharides would be substrates of T. forsythia AmpG. Thus, we produced defined fragments of $E$. coli PGN by controlled enzymatic digestion and provided these as MurNAc substitutes for growth of $T$. forsythia wild-type versus $\Delta a m p G$. While growth of the wild-type was unrestrictedly sustained when cultivated with GlcNAc-MurNAc(-peptides) and only slightly growth-impaired with GlcNAc-anhMurNAc(-peptides), T. forsythia $\triangle a m p G$ was completely growth inhibited in either case (Fig. 1d). Additionally, both disaccharides GlcNAc-anhMurNAc and GlcNAc-MurNAc - were detected in culture supernatants of T. forsythia $\triangle a m p G$ (Fig. 5), comparable to the situation found in E. coli $\triangle a m p G$, that, however, releases only GlcNAc-anhMurNAc. Regarding the importance of peptides being attached to the disaccharides, no difference in growth of $T$. forsythia wild-type was observed when cultivated with disaccharide-peptides or 


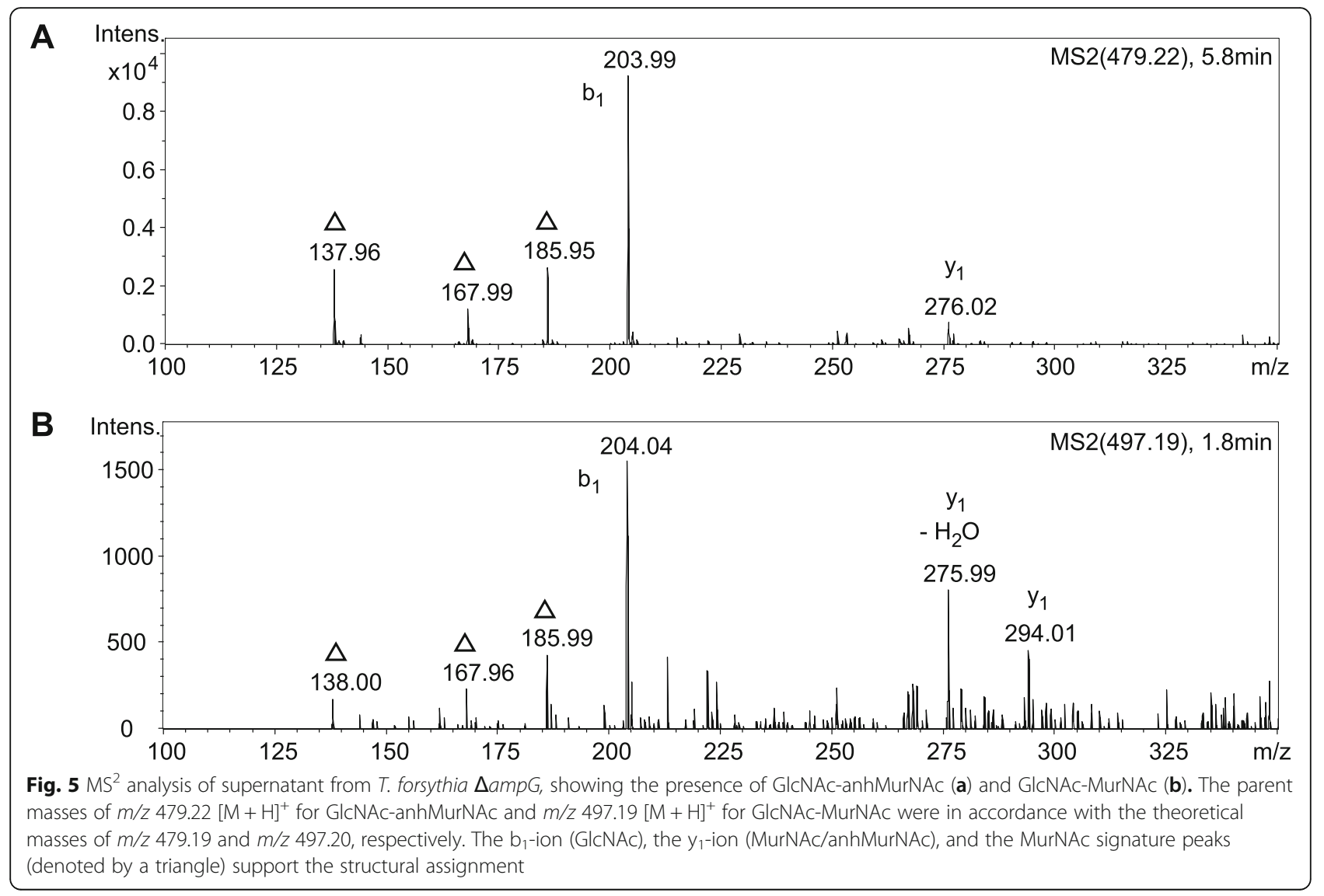

disaccharides alone. However, the fact that T. forsythia cell extracts exclusively produce disaccharide digestion products and T. forsythia $\triangle a m p G$ releases only disaccharides into the medium, might indicate the presence of a strong amidase activity in the periplasm of T. forsythia. That $T$. forsythia AmpG is also able to take up disaccharides carrying stem peptides might be concluded from the study by Ruscitto et al. [11], where the cytosolic accumulation of anhMurNAc-peptides in an E. coli double mutant lacking the muropeptide transporter AmpG and the amidase AmpD in comparison to the E. coli strain complemented with T. forsythia ampG indicated that AmpG transported E. coli's main PGN recycling product GlcNAcanhMurNAc-peptides into the cytoplasm. The usability of muropeptides as a growth factor by $T$. forsythia was supported by the authors of that study by demonstrating that a PGN digest of $F$. nucleatum could sustain bacterial growth. However, the exact composition of these muropeptides remained elusive since the interpretation of the corresponding MS data was based on the presence of $m$-Dap, which, however, is not a constituent of $F$. nucleatum PGN [17].

\section{Conclusion}

The inner membrane transporter AmpG of the periodontal pathogen $T$. forsythia is essential for growth of the bacterium on complex MurNAc sources provided by cohabiting bacteria in the oral cavity and imports both the long-standing substrate GlcNAc-anhMurNAc as well as GlcNAc-MurNAc into the cytoplasm. The capacity or need for also metabolizing GlcNAc-MurNAc(-peptides) might be explained by $T$. forsythia's MurNAc-auxotrophy. An important question that is currently addressed in our laboratories concerns how the PGN fragments that are imported via AmpG are processed in T. forsythia.

This study increases our understanding of the PGN metabolism of $T$. forsythia and simultaneously might reveal a new perspective on AmpG permeases, for which the GlcNAc-1,6-anhydroMurNAc disaccharide has been regarded as a minimum substrate signature in many Gram-negative bacteria. The new finding of this study of also GlcNAc-MurNAc being a growth factor of $T$. forsythia and AmpG substrate might have future implications for AmpG as a potential target for resistanceattenuating therapeutics [36].

\section{Methods}

Bacterial strains, growth conditions and growth curves Tannerella forsythia ATCC 43037 type strain (T. forsythia wild-type), T. forsythia ATCC $43037 \Delta a m p G$ (TfampG::ermF; for the construction of the mutant see next 
Table 1 Oligonucleotide primers used for PCR amplification reactions. Primers for qPCR are shaded in grey

\begin{tabular}{|c|c|}
\hline Primer & Sequence $\left(5^{\prime}-3^{\prime}\right)$ \\
\hline 460 & ATGACAAAAAAGAAATTGCCCGTTCGTTTTAC \\
\hline 461 & CTACGAAGGATGAAATTTTTCAGGGACAAC \\
\hline 524 & GTAAAACGAACGGGCAATTTCTTTTTTTGTCAT \\
\hline 525 & CCCTGAAAAATTTCATCCTTCGTAG \\
\hline 634 & AGTGCTACGGCTTCGCTTGAG \\
\hline 635 & GAACGGGCAATTTCTTTTTTGTCATTTGTTGTTTGTTTTAGTTAATC \\
\hline 636 & CCCTGAAAAATTTCATCCTTCGTAGTCGACGGATGAAACGGATTCATATC \\
\hline 637 & TGCCGATAGTCGGTGATATGC \\
\hline 638 & GACCGGTATCAATGTGATCG \\
\hline 639 & CCGCGATTTCCTGCATAACG \\
\hline 640 & TACTTTGCGGAAGGACTGC \\
\hline 641 & GCCGAGAGGTACACGTAGAG \\
\hline RecA for & AGCCGTCAACACAGACAAAC \\
\hline RecA rev & AGCCGGTAGGAATGACTTCG \\
\hline 16SrRNA for & TTTCAAGCCCGGAACGACAG \\
\hline 16SrRNA rev & CCGATACCGTCGATCAAATG \\
\hline AmpG for & CGGCCATCTTTATCGGACTG \\
\hline AmpG rev & ACGACGTGAATGTACGGAAG \\
\hline
\end{tabular}

paragraph), Fusobacterium nucleatum subsp. nucleatum OMZ 598, and Porphyromonas gingivalis W83 were cultivated anaerobically at $37^{\circ} \mathrm{C}$ in brain heart infusion medium (BHI; $37 \mathrm{~g} \mathrm{l}^{-1}$; Oxoid, Basingstoke, United Kingdom), supplemented with yeast extract ( $10 \mathrm{gl}^{-1}$; Sigma, Vienna, Austria), L-cysteine $\left(1 \mathrm{gl}^{-1}\right.$; Sigma), hemin $\left(5 \mu \mathrm{g} \mathrm{ml}^{-1}\right.$; Sigma), and menadione $\left(2 \mu \mathrm{g} \mathrm{ml}^{-1}\right.$; Sigma). For T. forsythia strains, $5 \%(\mathrm{v}$ $\mathrm{v}^{-1}$ ) horse serum (Thermo Fisher Scientific, Vienna, Austria) and MurNAc $\left(10 \mu \mathrm{g} \mathrm{ml}^{-1}\right.$ or $\left.1 \mu \mathrm{g} \mathrm{ml}^{-1}\right)$ Carbosynth, Compton, United Kingdom) were added, if not stated otherwise; $T$. forsythia ATCC $43037 \triangle a m p G$ was grown with additional supplementation of $5 \mu \mathrm{g} \mathrm{ml}^{-1}$ erythromycin. Escherichia coli MC4100 and the E. coli TP72 (F- lysA opp araD139 rpsL150 relA1 deoC1 ptsF25 ftbB5301 rbsR_(argF-lac) ampG::Kan) [37] were routinely cultivated in Miller's LB broth base supplemented with $50 \mu \mathrm{g} \mathrm{ml}^{-1}$ kanamycin (Thermo Fisher Scientific) at $37^{\circ} \mathrm{C}$ with constant shaking. For the preparation of MurNAc substitutes from E. coli, BHI medium was used instead of Miller's LB broth base for comparability.

For recording of growth curves, $10 \mathrm{ml}$ of bacterial cultures were inoculated to a starting optical density at 600 $\mathrm{nm}\left(\mathrm{OD}_{600}\right)$ of 0.05 and biological triplicates were measured in 1-ml cuvettes with a cell density meter (Ultraspec 10; Amersham Biosciences, Austria) at several time points until the stationary phase was reached. Samples of $\mathrm{OD}_{600}<0.7$ were measured directly, while samples of $\mathrm{OD}_{600}>0.7$, were diluted by $1: 10$.

\section{Construction of a $T$. forsythia $\triangle a m p G$ deletion mutant}

The gene encoding the AmpG muropeptide transporter homologue (Tanf_08365) was deleted by homologous recombination using a knock-out vector, replacing the ampG gene by an erythromycin resistance marker gene (Supplemental Fig. S6). A detailed description of the construction of a deletion mutant in T. forsythia is published elsewhere [38]. Briefly, a knock-out cassette was constructed by PCR amplification of $1 \mathrm{kbp}$-long homologous up- and downstream regions of the ampG gene, flanking the ermF marker gene, using Phusion HighFidelity DNA polymerase (Thermo Fisher Scientific). Up- and downstream homologous regions were amplified from $T$. forsythia genomic DNA by primer pairs 634/635 and 636/637, respectively, and the ermF gene was amplified from vector pJET/dTF0955ko [38] using primer pair 460/461 [39] (Table 1). The final knock-out cassette was produced by overlap extension PCR and blunt-end cloned into the cloning vector pJET1.2 (Thermo Fisher Scientific). Approximately $5 \mu \mathrm{g}$ of the final knock-out vector pJET1.2/DTanf_08365 were transformed into $100 \mu \mathrm{l}$ of $T$. forsythia cells by electroporation and viable clones were selected on $\mathrm{BHI}$ agar plates containing erythromycin $\left(5 \mu \mathrm{g} \mathrm{ml}^{-1}\right)$. The correct integration of the knock-out cassette was tested by screening PCR using primer pairs 638/524 and 525/639 (Supplemental Fig. S6, Table 1). 


\section{Preparation of MurNAc sources}

Three types of MurNAc substitutes were prepared, including bacterial culture supernatants, intact PGN sacculi, and defined PGN fragments.

Culture supernatants were harvested by centrifugation (8000 g, $10 \mathrm{~min}$ ) from $50 \mathrm{ml}$ of P. gingivalis, F. nucleatum, and E. coli MC4100 and TP72 ( $\triangle a m p G)$ cultures at $\mathrm{OD}_{600} \sim 2.0$ (corresponding to the exponential growth phase), sterile-filtered, and concentrated seven-fold, using a SpeedVac vacuum centrifuge (Thermo Fisher Scientific). For growth experiments, $1 \mathrm{ml}$ of concentrated supernatant, each, was added to $10 \mathrm{ml}$ of $T$. forsythia culture.

PGN sacculi from T. forsythia, P. gingivalis, F. nucleatum, and E. coli were isolated following a published protocol $[16,26]$. In short, biomass was harvested from bacterial cultures from the stationary growth phase and boiled in 8\% SDS to lyse cells, followed by several washing steps with distilled water using ultracentrifugation $(60,000$ g, $30 \mathrm{~min}, 60^{\circ} \mathrm{C}$ ) and dialysis against distilled water. Subsequently, PGN preparations were treated with $\alpha$-amylase (24 mg ml${ }^{-1}$; Sigma) for $2 \mathrm{~h}$ at $37^{\circ} \mathrm{C}$ and, subsequently, with pronase $\left(10 \mathrm{mg} \mathrm{ml}^{-1}\right.$; Sigma) for $2 \mathrm{~h}$ at $60^{\circ} \mathrm{C}$ to degrade contaminating carbohydrates and proteins, boiled, washed, and dried in a SpeedVac vacuum centrifuge [16].

To correct for differences in purity, the MurNAc concentration in the different bacterial culture supernatants and PGN preparations was determined by HPAEC-PAD. For this purpose, seven-fold concentrated bacterial culture supernatants and $400 \mu \mathrm{g}$ of PGN, respectively, were hydrolysed with $400 \mu \mathrm{l}$ of $6 \mathrm{~N} \mathrm{HCl}$ containing $0.2 \%$ thioglycolic acid and incubated at $110^{\circ} \mathrm{C}$ over-night. Samples were dried, washed, resuspended in $120 \mu \mathrm{l}$ of distilled water and sterile-filtered, before $20 \mu \mathrm{l}$ of material were injected into the HPAEC-PAD system ICS3000, using a CarboPac PA1 column (Dionex, Thermo Fisher Scientific) and applying a flow rate of $1 \mathrm{ml} \mathrm{min}{ }^{-1}$. Separation of sugar constituents was performed starting with an isocratic elution of $15 \%$ solvent A ( $100 \mathrm{mM}$ sodium hydroxide in distilled water) and $0.2 \%$ solvent $B$ ( $1 \mathrm{M}$ sodium acetate in solvent A) for $21 \mathrm{~min}$, followed by linear gradients to $98.8 \% \mathrm{~A}$ and $0.2 \% \mathrm{~B}$ until min 27 , changing to $68 \%$ $\mathrm{A}$ and $32 \% \mathrm{~B}$ until min 59, back to $98.8 \% \mathrm{~A}$ and $0.2 \% \mathrm{~B}$ until min 65 and to $15 \%$ A and $0.2 \%$ B until min 68, maintained until min 80 . For growth experiments, PGN isolates were added as a medium component, equalling a MurNAc concentration of $\sim 1 \mu \mathrm{g} \mathrm{ml}^{-1}$.

To obtain defined PGN fragments, $3 \mathrm{mg}$ of E. coli PGN was digested with Streptomyces globisporus $N$ acetylmuramidase mutanolysin $\left(50 \mu \mathrm{g} \mathrm{ml}^{-1}\right.$; Sigma $)$ or E. coli lytic transglycosylase Slt70 $\left(100 \mu \mathrm{g} \mathrm{ml}^{-1}\right)$ [40, 41], dissolved in $10 \mathrm{mM}$ HEPES buffer containing $0.1 \mathrm{M}$ $\mathrm{NaCl}$ and $0.1 \%$ Triton $^{\text {Tx }} \mathrm{X}-100$ (Sigma), in a final volume of $420 \mu \mathrm{l}$. For digestion with E. coli amidase AmiD $\left(50 \mu \mathrm{g} \mathrm{ml}^{-1}\right)$ [25], $5 \mu \mathrm{M}$ zinc chloride (Sigma) was added. Incubation was performed at $37{ }^{\circ} \mathrm{C}$ under constant shaking overnight and reactions were stopped by heating at $100{ }^{\circ} \mathrm{C}$ for $25 \mathrm{~min}$. These specific digests were used as MurNAc substitutes, applying the same concentrations as for undigested PGN.

Slt70 and AmiD were produced as recombinant, $\mathrm{His}_{6}$-tagged enzymes in E. coli BL21 (DE3) cells carrying the expression plasmid pET29b-slt70 and pET28aEc_amiD, respectively. Transformed E. coli was grown in $400 \mathrm{ml}$ of LB broth supplemented with kanamycin $\left(50 \mu \mathrm{g} \mathrm{ml}^{-1}\right)$ at $37^{\circ} \mathrm{C}$ and $200 \mathrm{rpm}$. At the midexponential growth phase $\left(\mathrm{OD}_{600} \sim 0.6\right)$, protein expression was induced with $1 \mathrm{mM}$ isopropyl- $\beta$-D-thiogalactopyranosid (IPTG) and cultivation was continued for $4 \mathrm{~h}$. Cells were harvested by centrifugation $(4500 \mathrm{~g}, 20$ min, $4{ }^{\circ} \mathrm{C}$ ). Purification of $\mathrm{His}_{6}$-tagged enzymes followed a previously established protocol [34]. Briefly, cell pellets were resuspended in lysis buffer $(50 \mathrm{mM}$ Tris- $\mathrm{HCl}, \mathrm{pH} 7.6,300 \mathrm{mM} \mathrm{NaCl}$ ) containing $10 \mathrm{mM}$ imidazole and EDTA-free protease inhibitor mixture (cOmplete, Roche Applied Science), followed by ultrasonication. Lysates were centrifuged $(40,000 \mathrm{~g}, 40 \mathrm{~min}$, $4{ }^{\circ} \mathrm{C}$ ) and the supernatant fraction was applied to a nickel-nitrilotriacetic acid (Qiagen) affinity chromatography column equilibrated in lysis buffer. After washing the column with 10 column volumes of washing buffer (50 mM Tris- $\mathrm{HCl}, \mathrm{pH} 7.6,300 \mathrm{mM} \mathrm{NaCl}, 30 \mathrm{mM}$ imidazole), the $\mathrm{His}_{6}$-tagged protein was eluted with $200 \mathrm{mM}$ imidazole. Fractions containing the purified enzyme, as determined by SDS-PAGE (12\% gel) upon Coomassie Brilliant Blue G250 (CBB) staining, were pooled and dialyzed against dialysis buffer $(50 \mathrm{mM}$ Tris- $\mathrm{HCl}, \mathrm{pH} 7.6$, $100 \mathrm{mM} \mathrm{NaCl}$ ) overnight at $4{ }^{\circ} \mathrm{C}$. Protein concentration was measured spectrophotometrically and calculated using $\mathrm{A}_{280} 0.1 \%$ values obtained from the exPASy ProtParam tool (http://web.expasy.org/protparam).

\section{Analysis of culture supernatants}

One hundred $\mu \mathrm{l}$ of bacterial culture supernatants from $\mathrm{OD}_{600} \sim 2.0$-cultures (as described above) were harvested by centrifugation $(8000 \mathrm{~g}, 15 \mathrm{~min})$ and prefiltered over a Durapore $^{\text {Tu }} 0.22 \mu \mathrm{m}$ cartridge (Merck, Vienna, Austria). Subsequently, samples were dried in a SpeedVac vacuum centrifuge (Thermo Fisher Scientific, Vienna, Austria) and dissolved in $15 \mu \mathrm{l}$ of $80 \mathrm{mM}$ formate, buffered to $\mathrm{pH} 3.5$ with ammonia (solvent A). In the case of $P$. gingivalis, the supernatant was enriched via HILIC-SPE (2.5 g microcrystalline cellulose; Merck), bringing $100 \mu$ of supernatant to a total volume of $2 \mathrm{ml}$, matching the starting conditions of the self-packed column ( $90 \%$ acetonitrile in $0.1 \%$ trifluoroacetic acid (TFA)), followed by washing with $10 \mathrm{ml} 90 \%$ acetonitrile in $0.1 \%$ TFA and elution with $5 \mathrm{ml}$ of $40 \%$ acetonitrile in $0.1 \%$ TFA. Separation was performed with a Kinetex XB-C18 column $(150 \times 2.1 \mathrm{~mm}, 100 \AA ̊, 1.7 \mu \mathrm{m}$; 
Phenomenex) on a Nexera X2 HPLC (Shimadzu, Korneuburg, Austria) connected to an amaZon ion trap mass spectrometer (Bruker, Bremen, Germany). At a flow rate of $0.25 \mathrm{ml} \mathrm{min}^{-1}$ and a column oven temperature of $42{ }^{\circ} \mathrm{C}$, an initial concentration of $2 \%$ solvent B ( $80 \%$ acetonitrile in solvent A) was held for 5 min before applying a linear gradient to $10 \%$ B over 15 min. Percentage B was further raised to 35 and $95 \%$ over 5 and $15 \mathrm{~min}$, respectively. Operation of the ion trap was done in the positive ion mode with DDA (Capillary $4.5 \mathrm{kV}$ ). Data was analysed in PostrunAnalysis with LabSolutions 5.73 (Shimadzu, Germany) and Bruker Daltonics DataAnalysis 4.0.

\section{Analysis of peptidoglycan fragments}

PGN fragments produced by digestion of E. coli PGN with mutanolysin, Slt70, and AmiD, respectively, were analysed by LC-ESI-MS, as described above under "Analysis of culture supernatants".

PGN-type analysis of $F$. nucleatum was performed following a recently published protocol [16]. Briefly, PGN was resuspended in $200 \mathrm{mM}$ sodium phosphate buffer, $\mathrm{pH}$ 6.0, digested with S. globisporus mutanolysin $\left(50 \mu \mathrm{g} \mathrm{ml}^{-1}\right.$; Sigma) overnight at $37^{\circ} \mathrm{C}$ and reduced with sodium borohydride. The supernatant was dried in a SpeedVac vacuum centrifuge (Thermo Fisher Scientific) and preparations were applied to HPLC, as published elsewhere [42]. LC-ESI-MS measurements were performed using a $\mathrm{C} 18$ Gemini column $(150 \times 4.6 \mathrm{~mm}, 110 \AA$, $5 \mu \mathrm{m}$; Phenomenex $)$ and an UltiMate 3000 HPLC system (Dionex) coupled to a MicrOTOF II mass spectrometer (Bruker), operated in positive ion mode [42].

\section{Synthesis of anhydro- $\mathrm{N}$-acetylmuramic acid}

To serve as a standard for the analysis of the different MurNAc sources investigated within the frame of this study and as a putative growth supplement for $T$. forsythia, anhMurNAc was chemically synthesized via a fivestep synthesis scheme according to a published protocol [29], with minor modifications. For the introduction of the trityl group at position four during the third step, the formation of the reagent trityl triflate beforehand is necessary. This step revealed to be very temperature sensitive and only worked, if the addition of tritylalcohol to a solution of trimethylsilyl trifluoromethanesulfonate was carried out at low temperature $\left(-78^{\circ} \mathrm{C}\right)$. Once trityl triflate had been formed, it could be used for the introduction of the trityl group also at moderate temperatures $\left(0^{\circ} \mathrm{C}\right)$.

The purification of the final deprotected anhMurNAc was achieved by HILIC. Crude deprotected anhMurNAc was dissolved in a minimal amount of water and injected via a sample loop into a semi-preparative HILIC column (SeQuant ZIC HILIC column -Merck, $25 \times 1 \mathrm{~cm}$, Cat No 1.50494.0001) connected to an Interchim 4125 ELSD preparative HPLC system. A gradient from 100\% acetonitrile to $40 \%$ acetonitrile and $60 \% 5 \mathrm{mM}$ ammonium acetate over 10 column volumes was employed. Elution was monitored by ELSD, product-containing fractions were collected, dried in a SpeedVac centrifuge and checked individually by NMR spectroscopy.

NMR spectra were recorded at $297 \mathrm{~K}$ in $99.9 \%$ acetonitrile with a Bruker Avance III 600 spectrometer $\left({ }^{1} \mathrm{H}\right.$ at $600.13 \mathrm{MHz},{ }^{13} \mathrm{C}$ at $150.9 \mathrm{MHz}$ ), using standard Bruker NMR software. ${ }^{1} \mathrm{H}$ NMR were referenced internally to the residual solvent signal $(1.94 \mathrm{ppm})$. Care needed to be taken upon analysing anhMurNAc by NMR, as only the protonated form is soluble in acetonitrile, for which the shifts have been reported [29] and could be reproduced. The chemical shifts in other solvents such as MeOD differed greatly, even from fraction to fraction (this study). Therefore, fractions after HILIC purification were acidified to a $\mathrm{pH}$ of 4.0 (Dowex 50XW8) before further processing.

In growth experiments, anhMurNAc was added at a concentration of $10 \mu \mathrm{g} \mathrm{ml}^{-1}$ to substitute MurNAc.

\section{Digestion of peptidoglycan by $T$. forsythia cell extracts}

To determine an overall PGN degrading activity of soluble $T$. forsythia enzymes, three biological replicates of $T$. forsythia wild-type supplemented with free MurNAc $(10 \mu \mathrm{g}$ $\mathrm{ml}^{-1}$ ) or E. coli PGN were cultivated as described above. When an $\mathrm{OD}_{600}$ of 0.8 had been reached, $30 \mathrm{ml}$ of $T$. forsythia culture were harvested by centrifugation $(8000 \mathrm{~g}$, $10 \mathrm{~min}, 4^{\circ} \mathrm{C}$ ). Cells were washed, resuspended in $1.5 \mathrm{ml}$ of $10 \mathrm{mM}$ Tris- $\mathrm{HCl}, \mathrm{pH} 8.0$, and lysed by ultrasonication $(4 \times 30 \mathrm{~s}$, on ice). After centrifugation $(14,000 \mathrm{~g}, 15 \mathrm{~min}$, $4{ }^{\circ} \mathrm{C}$ ), the enzyme-rich supernatant was incubated with $0.5 \mathrm{mg}$ of $E$. coli $\mathrm{PGN}$ at $37^{\circ} \mathrm{C}$. Samples were prepared after $0,1,5$ and $20 \mathrm{~h}$ by mixing $300 \mu \mathrm{l}$ of incubation solution with $1.2 \mathrm{ml}$ of ice-cold acetone followed by incubation for $15 \mathrm{~min}$ at $4{ }^{\circ} \mathrm{C}$. After centrifugation $(12,000 \mathrm{~g}, 10 \mathrm{~min}$, $\left.4{ }^{\circ} \mathrm{C}\right)$, the supernatant was dried in a SpeedVac vacuum centrifuge (Thermo Fisher Scientific) and resuspended in $100 \mu \mathrm{l}$ of distilled water. Five-microliters of each sample were analysed by LC-ESI-MS operated in negative ion mode, applying a 45-min HPLC program (for $5 \mathrm{~min}, 100 \%$ buffer A: $0.1 \%$ formic acid with $0.05 \%$ ammonium formate, then $30 \mathrm{~min}$ of a linear gradient to $40 \%$ buffer B $\left(\mathrm{CH}_{3} \mathrm{CN}\right)$, and $10 \mathrm{~min}$ of $100 \%$ buffer A for column reequilibration) according to [43], including an optimized column re-equilibration step. Exact masses for MurNAc, GlcNAc-MurNAc, GlcNAc-anhMurNAc and anhMurNAc, were presented as extracted ion chromatograms using Data Analysis 4 (Bruker), and the peak areas under the curve were quantified with GraphPad Prism 6.

To analyse disaccharides consisting of GlcNAc and MurNAc, samples were further digested with $50 \mu \mathrm{g} \mathrm{ml}^{-1}$ of Bacillus subtilis $\mathrm{N}$-acetylglucosaminidase NagZ [43] (available in the laboratory of C. Mayer) for $4 \mathrm{~h}$ at $37^{\circ} \mathrm{C}$. Five-microliters of each sample were analysed 
immediately by LS-MS run in negative ion mode as described previously [44] in order to ensure the efficient separation of GlcNAc-MurNAc and MurNAc on a SeQuant ${ }^{\circ}$ ZIC $^{\oplus}$-pHILIC column (Merck, PEEK $150 \times 2.1$ $\mathrm{mm}, 5 \mu \mathrm{m})$.

\section{Determination of the relative expression level of AmpG by qPCR}

To determine, if $\operatorname{amp} G$ was upregulated upon growth with PGN as a MurNAc source, three biological replicates of $T$. forsythia wild-type were cultivated upon supplementation with $E$. coli PGN (corresponding to a MurNAc concentration of $1 \mu \mathrm{g} \mathrm{ml}^{-1}$ ) and, for comparison, free MurNAc, as described above, and $10 \mathrm{ml}$ of culture from the exponential phase and $5 \mathrm{ml}$ of culture from the stationary phase, respectively, were harvested by centrifugation $(8000 \mathrm{~g}, 10$ min). RNA was isolated using PureLink ${ }^{\text {Tx }}$ RNA Mini Kit (Thermo Fisher Scientific) with TRIzol $^{\mathrm{Ts}}$ reagent as described by the manufacturer, including an on-column PureLink $^{\mathrm{Tm}}$ DNAase (Thermo Fisher Scientific) treatment. Fifty ng of RNA were transcribed to cDNA, using the HighCapacity cDNA Reverse Transcription Kit (Thermo Fisher Scientific). For each cDNA sample, a reverse transcriptase control was included to test for genomic DNA contaminations. Standards were prepared for the target gene ampG and the house-keeping genes recA and 16SrRNA by PCR, using the Phusion High-Fidelity DNA Polymerase (Thermo Fisher Scientific), listed primers (Table 1), and genomic DNA of T. forsythia isolated according to a published protocol [45]. After verifying the specificity of primers by agarose gel electrophoresis $(1.5 \%, 80 \mathrm{~V}, 40 \mathrm{~min})$, PCR products were purified using the GeneJET Gel Extraction Kit (Thermo Fisher Scientific) and diluted in a series of 1:10steps to $10^{8}-10^{3}$ copies per $\mu \mathrm{l}$ for generating a standard curve. Quantitative real-time PCR was performed with gene specific primers (Table 1) on a Rotor-Gene Q real-time PCR cycler (Corbett Research, Qiagen, Germantown, MD, USA), using Power SYBR Green PCR Master Mix (Thermo Fisher Scientific). Reactions were set up in triplicate, including $5 \mu \mathrm{l}$ master mix, $1 \mu \mathrm{l}$ primer mix $(500 \mathrm{nM}), 3 \mu \mathrm{l}$ DEPCtreated water (Thermo Fisher Scientific), and $1 \mu \mathrm{l}$ cDNA. Relative expression levels of $a m p G$ were quantified using the relative standard curve method and normalized to the expression of house-keeping genes recA and 16S rRNA, using the geometric mean as described by Taylor et al. [46]. Statistically significant differences $(P<0.05)$ between groups were determined using the unpaired student's t-test.

\section{Scanning electron microscopy (SEM)}

T. forsythia wild-type and T. forsythia $\triangle a m p G$ were cultivated with free MurNAc or E. coli PGN, as described above, and samples were prepared for SEM following a published protocol [38]. In short, $1 \mathrm{ml}$ of each culture was harvested by centrifugation $(8000 \mathrm{~g}, 7 \mathrm{~min})$, cell pellets were washed with phosphate buffered saline and applied to increasing concentrations of ethanol from 25 to $100 \%$ in PBS, with incubation of $7 \mathrm{~min}$ and removal of ethanol by centrifugation after each step. Samples were sputtercoated with gold (EM SDC005 apparatus; Leica, Wetzlar, Germany) and imaged with an Inspect S50 scanning electron microscope (FEI, Eindhoven, The Netherlands).

\section{Supplementary information}

Supplementary information accompanies this paper at https://doi.org/10 1186/s12866-020-02006-z.

\section{Additional file 1.}

\section{Abbreviations}

Ala: Alanine; anhMurNAc: anhydro- $N$-acetylmuramic acid; $m$-DAP: mesodiaminopimelic acid; E. coli: Escherichia coli; ELSD: Evaporative light-scattering detection; F. nucleatum: Fusobacterium nucleatum; GlcNAc: N-

acetylglucosamine; Glu: Glutamic acid; HILIC: Hydrophobic interaction liquid chromatography; HPAEC-PAD: High-performance anion exchange chromatography with pulsed amperometric detection; IPTG: Isopropyl- $\beta-D-$ thiogalactopyranosid; LC-ESI-MS: Liquid chromatography-electrospray ionization-mass spectrometry; MeDO: Deuterated methanol; MS²: Tandem mass spectrometry; MurNAc: N-acetylmuramic acid; NMR: Nuclear magnetic resonance; $P$. gingivalis: Porphyromonas gingivalis; PGN: Peptidoglycan; qPCR: quantitative real-time PCR; RP-HPLC: Reversed phase high-performance liquid chromatography; S. globisporus: Streptomyces globisporus; SPE: Solid phase extraction; TFA: Trifluoroacetic acid; T. forsythia: Tannerella forsythia

\section{Acknowledgments}

Shahriar Mobashery and Ted Park are gratefully acknowledged for providing the expression plasmid for Slt70 and for AmiD, respectively. We thank Sonja Zayni for technical support with the determination of MurNAc/anhMurNAc concentrations by HPAEC-PAD.

\section{Authors' contributions}

VMTM, RF, MBo, CM, and CS conceptualized and planned experiments. VMTM, MBT, RF, MBo, $I H$ and MB performed the experiments. VMTM, MBT, $\mathrm{RF}, \mathrm{MBO}, \mathrm{H}, \mathrm{MB}, \mathrm{FA}, \mathrm{CM}$, and CS analysed the data. VMTM, CM, and CS wrote the manuscript and FA provided feedback on the manuscript. All authors read and approved the final manuscript.

\section{Funding}

This work was supported by the Austrian Science Fund (FWF), projects 12875 B22, P26836-B22 and P27374-B22 (to C.S.) and the German Research Foundation (DFG), project MA2436/7-1 (to C.M.). MB and IH acknowledge support from the DFG graduate school (GRK1708). The exclusive role of the funding bodies was to provide financial support for the study.

\section{Availability of data and materials}

The datasets used and/or analysed during the current study are available from the corresponding author on reasonable request.

Ethics approval and consent to participate

Not applicable.

\section{Consent for publication}

Not applicable.

\section{Competing interests}

The authors declare that they have no competing interests.

\section{Author details}

'Department of NanoBiotechnology, NanoGlycobiology unit, Universität für Bodenkultur Wien, Vienna, Austria. ${ }^{2}$ Department of Chemistry, Institute of Biochemistry, Universität für Bodenkultur Wien, Vienna, Austria. ${ }^{3}$ Department of Biology, Eberhard Karls Universität Tübingen, Microbiology/Glycobiology, 
Interfaculty Institute of Microbiology and Infection Medicine Tübingen, Tübingen, Germany. ${ }^{4}$ Department of Chemistry, Institute of Organic Chemistry, Universität für Bodenkultur Wien, Vienna, Austria.

Received: 27 May 2020 Accepted: 12 October 2020

Published online: 17 November 2020

\section{References}

1. Tanner ACR, Izard J. Tannerella forsythia, a periodontal pathogen entering the genomic era. Periodontol 2000. 2006;42:88-113.

2. Hajishengallis G. Periodontitis: from microbial immune subversion to systemic inflammation. Nat Rev Immunol. 2015;15(1):30-44.

3. Righolt AJ, Jevdjevic M, Marcenes W, Listl S. Global-, regional-, and country-level economic impacts of dental diseases in 2015. J Dent Res. 2018;97(5):501-7.

4. Cullinan M, Ford P, Seymour G. Periodontal disease and systemic health: current status. Aus dent J. 2009;54(s1):S62-9.

5. Dominy SS, Lynch C, Ermini F, Benedyk M, Marczyk A, Konradi A, Nguyen M, Haditsch U, Raha D, Griffin C, et al. Porphyromonas gingivalis in Alzheimer's disease brains: Evidence for disease causation and treatment with smallmolecule inhibitors. Sci Adv. 2019;5(1):eaau3333.

6. Socransky S, Haffajee A, Cugini M, Smith C, Kent R. Microbial complexes in subgingival plaque. J Clin Periodontol. 1998;25(2):134-44

7. Marsh PD, Zaura E. Dental biofilm: ecological interactions in health and disease. J Clin Periodontol. 2017;44(Suppl 18):S12-22.

8. Holt SC, Ebersole JL. Porphyromonas gingivalis, Treponema denticola, and Tannerella forsythia: the "red complex", a prototype polybacterial pathogenic consortium in periodontitis. Periodontol 2000. 2005;38:72-122.

9. Wyss C. Dependence of proliferation of Bacteroides forsythus on exogenous N-acetylmuramic acid. Infect Immun. 1989;57(6):1757-9.

10. Hottmann I, Mayer VMT, Tomek MB, Friedrich V, Calvert MB, Titz A, Schäffer C, Mayer C. N-Acetylmuramic acid (MurNAC) auxotrophy of the oral pathogen Tannerella forsythia: characterization of a MurNAc kinase and analysis of its role in cell wall metabolism. Front Microbiol. 2018;9:19.

11. Ruscitto A, Honma K, Veeramachineni VM, Nishikawa K, Stafford GP, Sharma A. Regulation and molecular basis of environmental muropeptide uptake and utilization in fastidious oral anaerobe Tannerella forsythia. Front Microbiol. 2017:8:648.

12. Friedrich $V$, Pabinger $S, C$ hen $T$, Messner $P$, Dewhirst FE, Schäffer C. Draft genome sequence of Tannerella forsythia type strain ATCC 43037. Genome Announc. 2015;3(3):e00660-15.

13. Vollmer W, Bertsche U. Murein (peptidoglycan) structure, architecture and biosynthesis in Escherichia coli. Biochim Biophys Acta. 2008;1778(9):1714-34.

14. Vollmer W, Seligman SJ. Architecture of peptidoglycan: more data and more models. Trends Microbiol. 2010;18(2):59-66.

15. Schleifer $\mathrm{KH}$, Kandler O. Peptidoglycan types of bacterial cell walls and their taxonomic implications. Bacteriol Rev. 1972;36(4):407-77.

16. Mayer VMT, Hottmann I, Figl R, Altmann F, Mayer C, Schäffer C. Peptidoglycan-type analysis of the $\mathrm{N}$-acetylmuramic acid auxotrophic oral pathogen Tannerella forsythia and reclassification of the peptidoglycan-type of Porphyromonas gingivalis. BMC Microbiol. 2019;19(1):200.

17. Kato K, Umenotoh T, Sagawa H, Kotani S. Lanthionine as an essential constituent of cell wall peptidoglycan of Fusobacterium nucleatum. Curr Microbiol. 1979:3:147-51.

18. Johnson JW, Fisher JF, Mobashery S. Bacterial cell-wall recycling. Ann N Y Acad Sci. 2013;1277:54-75

19. Bui NK, Gray J, Schwarz H, Schumann P, Blanot D, Vollmer W. The peptidoglycan sacculus of Myxococcus xanthus has unusual structural features and is degraded during glycerol-induced myxospore development. J Bacteriol. 2009;191(2):494-505.

20. Vötsch W, Templin MF. Characterization of a beta $N$-acetylglucosaminidase of Escherichia coli and elucidation of its role in muropeptide recycling and beta-actamase induction. J Biol Chem. 2000;275(50):39032-8.

21. Mayer C, Kluj RM, Mühleck M, Walter A, Unsleber S, Hottmann I, Borisova M. Bacteria's different ways to recycle their own cell wall. Int J Med Microbiol. 2019;309(7):151326.

22. Cheng Q, Park JT. Substrate specificity of the AmpG permease required for recycling of cell wall anhydro-muropeptides. J Bacteriol. 2002;184(23):6434-6.

23. Ruscitto A, Hottmann I, Stafford GP, Schäffer C, Mayer C, Sharma A. Identification of a novel $\mathrm{N}$-acetylmuramic acid transporter in Tannerella forsythia. J Bacteriol. 2016;198(22):3119-25.
24. Niwa D, Nishikawa K, Nakamura H. A hybrid two-component system of Tannerella forsythia affects autoaggregation and post-translational modification of surface proteins. FEMS Microbiol Lett. 2011;318(2):189-96.

25. Uehara T, Park JT. An anhydro-N-acetylmuramyl-L-alanine amidase with broad specificity tethered to the outer membrane of Escherichia coli. J Bacteriol. 2007;189(15):5634-41.

26. Desmarais SM, Cava F, de Pedro MA, Huang KC. Isolation and preparation of bacterial cell walls for compositional analysis by ultra performance liquid chromatography. J Vis Exp. 2014;83:e51183.

27. Vasstrand EN, Hofstad T, Endresen C, Jensen HB. Demonstration of lanthionine as a natural constituent of the peptidoglycan of Fusobacterium nucleatum. Infect Immun. 1979;25(3):775-80

28. Vasstrand EN, Jensen HB, Miron T, Hofstad T. Composition of peptidoglycans in Bacteroidaceae: determination and distribution of lanthionine. Infect Immun. 1982;36(1):114-22.

29. Calvert MB, Mayer C, Titz A. An efficient synthesis of 1,6-anhydro-N-acetylmuramic acid from N-acetylglucosamine. Beilstein J Org Chem. 2017;13:2631-6.

30. Lamont RJ, Hajishengallis G. Polymicrobial synergy and dysbiosis in inflammatory disease. Trends Mol Med. 2015:21(3):172-83.

31. Kolenbrander PE. Multispecies communities: interspecies interactions influence growth on saliva as sole nutritional source. Int J Oral Sci. 2011;3(2):49-54.

32. Uehara T, Suefuji $K$, Valbuena N, Meehan B, Donegan M, Park JT. Recycling of the anhydro- $N$-acetylmuramic acid derived from cell wall murein involves a two-step conversion to N-acetylglucosamine-phosphate. J Bacteriol. 2005;187(11):3643-9.

33. Adin DM, Engle JT, Goldman WE, McFall-Ngai MJ, Stabb EV. Mutations in ampG and lytic transglycosylase genes affect the net release of peptidoglycan monomers from Vibrio fischeri. J Bacteriol. 2009;191(7):2012-22.

34. Garcia DL, Dillard JP. Mutations in ampG or ampD affect peptidoglycan fragment release from Neisseria gonorrhoeae. J Bacteriol. 2008;190(11):3799-807.

35. Zamorano L, Reeve TM, Juan C, Moya B, Cabot G, Vocadlo DJ, Mark BL, Oliver A. AmpG inactivation restores susceptibility of pan-beta-lactamresistant Pseudomonas aeruginosa clinical strains. Antimicrob Agents Chemother. 2011;55(5):1990-6.

36. Perley-Robertson GE, Yadav AK, Winogrodzki JL, Stubbs KA, Mark BL, Vocadlo DJ. A fluorescent transport assay enables studying AmpG permeases involved in peptidoglycan recycling and antibiotic resistance. ACS Chem Biol. 2016;11(9):2626-35.

37. Jacobs C, Huang LJ, Bartowsky E, Normark S, Park JT. Bacterial cell wall recycling provides cytosolic muropeptides as effectors for beta-lactamase induction. EMBO J. 1994;13(19):4684-94.

38. Tomek MB, Neumann L, Nimeth I, Koerdt A, Andesner P, Messner P, Mach L, Potempa JS, Schäffer C. The S-layer proteins of Tannerella forsythia are secreted via a type IX secretion system that is decoupled from protein 0 glycosylation. Mol Oral Microbio. 2014;29(6):307-20.

39. Honma K, Inagaki S, Okuda K, Kuramitsu HK, Sharma A. Role of a Tannerella forsythia exopolysaccharide synthesis operon in biofilm development. Microb Pathog. 2007:42(4):156-66.

40. Höltje JV, Mirelman D, Sharon N, Schwarz U. Novel type of murein transglycosylase in Escherichia coli. J Bacteriol. 1975;124(3):1067-76.

41. Vollmer W, Blanot D, de Pedro MA. Peptidoglycan structure and architecture. FEMS Microbiol Rev. 2008;32(2):149-67.

42. Borisova M, Gisin J, Mayer C. Blocking peptidoglycan recycling in Pseudomonas aeruginosa attenuates intrinsic resistance to fosfomycin. Microb Drug Resist. 2014;20(3):231-7.

43. Litzinger S, Duckworth A, Nitzsche K, Risinger C, Wittmann V, Mayer C. Muropeptide rescue in Bacillus subtilis involves sequential hydrolysis by $\mathrm{b}-\mathrm{N}$-acetylglucosaminidase and N-acetylmuramyl-L-alanine amidase. J Bacteriol. 2010;192(12):3132-43.

44. Kästle B, Geiger T, Gratani FL, Reisinger R, Goerke C, Borisova M, Mayer C, Wolz C. rRNA regulation during growth and under stringent conditions in Staphylococcus aureus. Environ Microbiol. 2015;17(11):4394-405.

45. Cheng HR, Jiang N. Extremely rapid extraction of DNA from bacteria and yeasts. Biotechnol Lett. 2006;28(1):55-9.

46. Taylor SC, Nadeau K, Abbasi M, Lachance C, Nguyen M, Fenrich J. The ultimate $\mathrm{qPCR}$ experiment: producing publication quality, reproducible data the first time. Trends Biotechnol. 2019;37(7):761-74.

\section{Publisher's Note}

Springer Nature remains neutral with regard to jurisdictional claims in published maps and institutional affiliations. 